The effects of rumination induction on attentional breadth for self-related information Maud Grol $^{1}$, Paula T. Hertel ${ }^{2}$, Ernst H.W. Koster ${ }^{1}$, Rudi De Raedt ${ }^{1}$

\author{
Author Note \\ ${ }^{1}$ Department of Experimental Clinical and Health Psychology, Ghent University, Belgium. \\ ${ }^{2}$ Department of Psychology, Trinity University, USA. \\ Correspondence concerning this article should be addressed to Maud Grol, \\ Department of Experimental Clinical and Health Psychology, Ghent University, Henri \\ Dunantlaan 2, B-9000, Ghent, Belgium. E-mail: Maud.Grol@UGent.be
}




\begin{abstract}
The attentional scope model of rumination describes the links between rumination and attentional breadth. The model postulates that a more narrow attentional scope, caused by negative mood, increases the likelihood that thoughts become repetitive on the same topic, which in turn could exacerbate negative mood and lead to more attentional narrowing. We experimentally tested this model by examining the attentional effects of rumination using a newly developed rumination- versus problem-solving induction. In the first experiment we found that only at high levels of trait rumination, induction of rumination compared to a problem-solving approach was associated with more attentional narrowing for self-related information relative to other-related information. A second experiment on the relationship between trait rumination and attentional breadth in the absence of induced rumination, revealed that especially trait brooding was related to more narrowed attention for self-related information relative to other-related information.
\end{abstract}

Keywords: rumination; attention; attentional breadth; self-related information 


\section{The effects of rumination induction on attentional breadth for self-related information}

Rumination can be defined as repetitive, intrusive, negative thoughts, and as a mode of responding to distress. Rumination has been related to depression and even predicts (new episodes of) depression (Nolen-Hoeksema, 2000). Chronic negative affect is related to heightened self-focus and especially a ruminative self-focus, suggesting a reciprocal relation (Mor \& Winquist, 2002). Indeed, it has been shown that self-focused rumination induction leads to more negative thinking and impairs interpersonal problem-solving in dysphoric people (Lyumbomirsky \& Nolen-Hoeksema, 1995). Many studies have also investigated the maladaptive consequences of rumination at the level of cognitive functioning. A recent review gives an overview of these research findings with regard to control-related processes and proposes an attentional scope model of rumination (Whitmer \& Gotlib 2013).

The attentional scope model of rumination postulates that a narrow attentional scope, caused by negative mood or low positive mood, will limit the activated thought-action repertoires. This increases the likelihood that thoughts become repetitive, for example biasing thoughts and attention to self-related information, and rumination is defined as repetitive thinking about one's feelings and problems (Nolen-Hoeksema, Wisco, \& Lyubomirski, 2008). In turn this ruminative focus could exacerbate negative mood leading to attentional narrowing and more repetitive thoughts, turning it into a vicious cycle (Whitmer \& Gotlib, 2013). This is consistent with several studies showing that negative emotions narrow people's attentional focus (e.g. Derryberry \& Tucker, 1994; Fredrickson \& Branigan, 2005). Furthermore, trait rumination has been related to spontaneous use of perspectives low in self-distance (Ayduk \& Kross, 2010). Whitmer and Gotlib (2013) therefore suggest that trait ruminators might have a more narrow attentional scope compared to non-ruminators.

The attentional scope model by Whitmer and Gotlib (2013) was based on a large number of studies which provided, however, mostly indirect evidence for attentional 
narrowing, because they mainly investigated working memory processes. Therefore, we aimed to further improve our understanding of the relation between rumination and attentional breadth. Based on research investigating the consequences of rumination on inhibition and working memory processes, rumination is believed to be characterized by narrow internal attention towards self-related themes (e.g. one's feelings and problems) (Whitmer \& Gotlib, 2013). Yet, such ruminative self-focused thinking may also influence attention to the external environment, especially in the presence of self-related information. Thus, ruminative thinking could lead to narrowed (external) visual attention, especially when confronted with selfrelated information, as this kind of information is congruent or conceptually associated with activated self-representations. In turn this could reduce the likelihood of thoughts shifting to another topic. However, the consequences of rumination on external, visual attentional processes remain under investigated. Therefore, we wanted to more directly test whether experimentally induced rumination causes a narrowing of visuospatial attentional breadth, especially when the target of attention is self-related. Additionally we tested whether trait rumination would be related to more narrowed attention, that is, in the absence of induced rumination, in line with predictions from the attentional scope model of rumination (Whitmer \& Gotlib, 2013).

We developed a new rumination induction and contrasted it to a problem-solving induction (focusing on what actions one should take) as problem-solving can also take the form of repeated thinking (Watkins, 2008) that occurs in a negative or stressful and selfrelevant context. However, ruminative self-focused thinking, that is, repetitive thinking in an abstract way about one's feelings and problems, is a less adaptive way of thinking in response to stress, whereas problem-solving is believed to be a more constructive and perhaps less perseverative way of thinking (Watkins, 2008). Although ruminative thinking and problemsolving can both occur in a stressful and self-relevant context, ruminative thinking might be 
related to a narrowing of attention, especially for self-related information, whereas problemsolving may require a more broad focus of attention in such a context. Therefore, we wanted to expose people to the same stressful, self-relevant scenario across both induction conditions but then manipulate how people think in response to such a situation. This allowed us to better control for the self-relevance and (negative) valence of the context across conditions, but then manipulate whether people think about this situation in a ruminative self-focused manner or in a more adaptive problem-solving manner. In this way we sought to reduce the likelihood of influences on attentional breadth for self-related information by factors other than the style of thinking in the context of a self-relevant stressful situation. Given the repetitive and selffocused nature of ruminative thinking we expected that in a stressful and self-relevant context, thinking in a ruminative self-focused manner as compared to thinking in a problem-solving manner would narrow attention especially (or relatively more) in the presence of self-related information as compared to other-related information.

In the first experiment we first investigated the effectiveness of our new developed rumination versus problem-solving induction on a measure of state rumination. We also assessed the effect of the induction on heart rate variability because previous research has shown that decreased heart rate variability is associated with the experience of stressful events and worry (Pieper, Brosschot, Van der Leeden, \& Thayer, 2007). Our main research question concerned the effect of the style of thinking in response to a stressful self-related event on visuospatial attentional breadth for self-related information compared to information that is not related to the self (other). We also examined whether trait rumination or the presence of depressive symptoms qualified the effect of induced rumination on attentional breadth in our paradigm. It could be that high levels of trait rumination and depressive symptoms are associated with stronger effects of induced rumination on attentional narrowing for selfrelated information, in keeping with the habitual mode of processing experienced by people 
who ruminate when they are sad. In a second study we tested whether trait rumination would be related to more narrowed attention for self-related information in comparison to otherrelated information.

To measure attentional breadth in response to self-versus-other information, we used a performance-based task which has been used before to measure fluctuations in attentional breadth related to centrally presented stimuli (Bosmans, Braet, Koster, \& De Raedt, 2009). A previous study in which the original paradigm was adjusted for our current purposes showed that an increase in positive mood was associated with attentional broadening for self-related information when contrasted to other-related information (Grol, Koster, Bruyneel, \& De Raedt, 2014). In the present study we hypothesized that inducing a ruminative way of thinking, reflecting a repetitive focus on the same topic over time, leads to a more narrowed attentional focus especially for self-related information as compared to other-related information. To investigate whether an effect of the rumination induction on attentional narrowing would be explained by changes in negative mood as proposed by Whitmer and Gotlib (2013), we also took into account changes in negative mood due to the induction.

\section{Experiment 1}

\section{Method}

Participants and design. Thirty-seven healthy undergraduates (32 females) aged between 18 and 27 years $(M=21.27, S D=2.01)$ participated in this experiment and were paid $€ 12$. They were randomly assigned to the rumination or problem-solving induction task. This experiment was approved by the local ethical committee of the Faculty of Psychology at Ghent University.

\section{Materials and tasks.}


Questionnaire measures. ${ }^{1}$ To check whether the rumination induction had worked, we administered the Momentary Ruminative Self-focus Inventory (MRSI; Mor, Marchetti, \& Koster, 2013) before and after the rumination induction. The MRSI is a six-item questionnaire measuring to what extent people are thinking in a ruminative self-focused manner "at this moment." An example item is: "Right now, I am thinking about the possible meaning of the way I feel." Participants rate this on a 7-point scale ranging from "totally not agree" to "totally agree." Additionally, we measured mood state before and after the induction procedure with the Dutch version of the Profile of Mood States (POMS; McNair, Lorr, \& Droppelman, 1971; Wald, 1984). The POMS consists of 32 descriptions of feelings and participants are asked to rate how the description fits their feeling "at this moment" on a scale ranging from 0 "absolutely not" to 4 "very much." From these 32 items, five subscales are calculated (Depression, Anger, Tension, Fatigue, and Vigor). The Depression subscale was of primary interest in this study.

Along with state rumination and mood state, we also assessed trait characteristics. We measured trait affectivity with the 20-item trait version of the Positive and Negative Affect Schedule (PANAS; Watson, Clark, \& Tellegen, 1988). Participants were asked to rate the degree to which they felt the emotions "in general" giving their ratings on a 5-point Likert scale ranging from 1 "very slightly" to 5 "very much." To measure the general tendency to ruminate, we administered the 22-item Ruminative Response Scale (RRS; Nolen-Hoeksema \& Morrow, 1991; Raes, Hermans, \& Eelen, 2003). Participants are instructed to indicate to what extent they think or do what is described in statements on a 4-point scale ranging from "almost never" to "almost always", when the participant is feeling “.. down, sad, or depressed." A total score and two subscales can be calculated, Reflection and Brooding, with

\footnotetext{
${ }^{1}$ We also measured trait resilience in all experiments, using the Dutch version of the Resilience Scale (RS-nl; Portzky, 2008) to examine the relationship between trait resilience and attentional breadth, but these analyses were explorative and not related to the hypotheses of the current study.
} 
the former representing a more adaptive form of rumination and the latter a more detrimental form (Treynor, Gonzalez, \& Nolen-Hoeksema, 2003). To assess the presence and severity of depressive symptoms, we used the 21-item Beck Depression Inventory (BDI-II-NL; Beck, Steer, \& Brown, 1996; Van der Does, 2002). The questionnaire consists of 21 statements (responses ranging from 0 to 3 ) and participants are asked to select the response that best describes the way the participant has been feeling during the past two weeks. Finally, trait anxiety was measured using the trait version of the State Trait Anxiety Inventory (STAI-trait; Spielberger, Gorsuch, Lushene, Vagge, \& Jacobs, 1983; Van der Ploeg, Defares, \& Spielberger, 2000). Participants are asked to rate how they feel "in general" on a 4-point scale ranging from "almost never" to "almost always."

Attentional breadth for self-related information. To measure attentional breadth for self-related information we used a variant of a previously developed attentional breadth task (Bosmans et al., 2009) that measures fluctations in attentional breadth related to centrally presented, personally relevant stimuli. We adjusted the task in such a way that attentional breadth is measured for centrally presented self-related contrasted to other-related information (Grol et al., 2014). The word "ME" (Dutch = IK) was used as self-related stimulus and "LR" was used as the other-related stimulus. Because of previous criticism about the use of "self" versus general "other" categories in implicit measures (e.g. Karpinski \& Steinman, 2006), for the other-related category we used a specific stimulus that does not refer to self. "LR" was described as the initials of an unknown-other participant who had already taken part in this study. None of the participants' initials were "LR."

In each trial of the experimental task a word appeared in the center of the screen, “ME” (Dutch: "IK”) or "LR.” Simultaneously with presentation of the central word, 16 gray

\footnotetext{
2 Using self versus more general "other" stimuli (e.g. ME, THEM) is problematic in that it becomes more difficult to infer that effects are driven by self-related stimuli or by the fact that other people in general (as a category) evoke attentional processes due to certain attitudes towards that category of other people. This is less likely with a non-defined but specific stimulus.
} 
dots with a diameter of $2 \mathrm{~cm}$ appeared around the word in two concentric circles. One circle appeared at $4.5 \mathrm{~cm}$ from the central word at $10^{\circ}$ of the visual angle, the other circle appeared at $11.2 \mathrm{~cm}$ from the central word at $25^{\circ}$ of the visual angle. The gray dots were arranged in pairs of two, one close and one far, situated on one of eight implicit axes. Simultaneously with presentation of the central stimulus and gray dots, a smaller, black circle with a diameter of $1.3 \mathrm{~cm}$ appeared in one of the gray dots, either close or far. The small black circle was the target stimulus that participants were asked to identify. After the simultaneous presentation of the stimuli, participants were asked to identify the central stimulus and to identify the axis on which the target stimulus (i.e. the smaller black circle) had appeared. Figure 1 illustrates a trial in this task.

The main dependent variable was the accuracy rate on the peripheral task (i.e. the proportion of correctly localized target stimuli) on trials in which participants also correctly identified the central word (to make sure participants maintained attention to the center of the screen during the task). Subsequently we calculated indices of Attentional Narrowing (ANI = accuracy when the black circle was close to the word minus accuracy when it was far from the word) for both ME trials and LR trials. This allowed us to calculate the ANI difference score $\left(\triangle \mathrm{ANI}=\mathrm{ANI}_{\mathrm{ME}}-\mathrm{ANI}_{\mathrm{LR}}\right)$ representing each individual's attentional breadth in response to presentation of self-related information contrasted with other-related information. Higher $\triangle \mathrm{ANI}$ scores reflect stronger attentional narrowing when the central word was self-related in comparison to when the central word was other-related.

The task consisted of eight practice trials with a presentation time of $250 \mathrm{~ms}$ to allow participants to get acquainted with the task, followed by eight practice trials with a presentation time of $68 \mathrm{~ms}$. In the test phase itself, stimuli were simultaneously presented for $68 \mathrm{~ms}$ in order to prevent confounds of saccadic eye movements in search of the peripheral target (Ball, Beard, Roenker, Miller, \& Griggs, 1988). The test phase consisted of 96 trials of 
four types: ME close, ME far, LR close, and LR far. They were randomly presented in two blocks of 48 trials.

Heart rate variability. Beat-to-beat heart rate was measured using a telemetric heartbeat monitor (Polar S810). Data were moderately filtered, a minimum zone of 6 beats a minute (Cottyn, De Clercq, Pannier, Crombez, \& Lennoir, 2006) with the Polar Precision Performance Software for Windows and further analyzed with Kubios. This is a specialized program in analyzing heart rate and heart rate variability (Niskanen, Tarvainen, Ranta-Aho, \& Karjalainen, 2004). We investigated heart rate variability (HRV) using the Root Mean Square of the Differences of Successive Intervals (RMSSD), based on the recommendations of the European Society of Cardiology and the North American Society of Pacing and Electrophysiology (1996). This index provides an indication of modulations in parasympathetic outflow (Kleiger, Stein, Bosner, \& Rottman, 1992), and it is recommended for short-term HRV analysis (Task Force, 1996).

Thinking style induction. We provided the following self-related scenario to all participants: The person is driving a car after a busy day. The person sits a little dazed behind the wheel and decides to put on some music. When he/she reaches for a CD from the glove box he/she suddenly hears and feels a load bang and the person is pressed hard back in the car seat. Startled and a little in shock the person gets out of the car and realizes that he/she hit a mother with two children on the bike. They are lying on the ground and it doesn't look good. Some of them seemed to have been seriously injured. ${ }^{3}$

Participants were asked to first vividly imagine the scenario, experiencing the event through their own eyes, from a first person perspective, as if they are driving the car that causes the accident. Participants were then instructed to think about this situation and its consequences either in a ruminative manner, or in a problem-solving manner. Participants in

${ }^{3}$ A previous, unpublished experiment with a similar design used a scenario with less emotional impact. In that experiment, no effect of the thinking-style manipulation was found. 
both conditions received 6 different sentences of instructions, given at once on the screen. Instructions in the rumination condition were for example "think about how guilty you feel" or "think about what consequences this has for how you see yourself." Instructions in the problem-solving condition were for example "think about which steps to take, a plan of action" or "think about which organizations could help you at this moment." Participants were asked to describe their thoughts and were instructed not to be concerned with the use of correct language or fluent sentences but to write their spontaneous thoughts as they occur. Instructions were given via a Word document on the computer and participants typed their thoughts, starting below the instructions.

Procedure. At the beginning of the experiment participants were asked whether they had recently been involved in a traffic accident; no participant was excluded on this basis. After informed consent, participants completed the trait questionnaires. No participant was excluded on the basis of a BDI-II-NL score greater than 29. Next, we put on the telemetric heartbeat monitor and measured a 20-min heart rate baseline in which participants were asked to relax. After this baseline period we continued beat-to-beat heart rate registration throughout the rest of the experiment, but the critical time period was during the thinking-style induction. After the baseline heart beat registration, the POMS and MRSI were administered. Then participants received either the rumination or problem-solving induction. A total of $10 \mathrm{~min}$ were allocated to both imagining the scenario and describing their thoughts. After the induction, the MRSI and POMS were administered again, followed by the attentional breadth task. For the attentional breadth task, participants were seated at a distance of $27 \mathrm{~cm}$ from a 19-in CRT-computer screen, using a chin rest to ensure correct positioning. At the end of the experiment, participants in the rumination condition also received the problem-solving induction for ethical reasons. Finally, participants were fully debriefed about the experiment. 


\section{Results}

Preliminary analyses. All trials on the attentional breadth task were deleted in which the central word was identified incorrectly, to ensure that participants were focusing on the center of the screen during the task; an average of $2.93 \%$ of the trials were deleted. Due to non-normality of the data, a Mann-Whitney nonparametric test was performed to test for differences between induction conditions. No induction-related differences were found in terms of the percentages of trials that were deleted, $U=169, p=.96, r=-.01$.

For the heart rate baseline data, we analyzed the data from only the last $10 \mathrm{~min}$ of the 20-min baseline period. This way, arousal due to starting to measure heart rate was excluded in the baseline. The duration of the baseline heart rate registration was of similar length as the heart rate registration during the thinking-style induction.

Participant characteristics. Means and standard deviations for scores on the questionnaires and mood measures are shown in Table 1. To test for pre-existing differences between the two experimental conditions, independent $t$-tests were performed on age, MRSI state rumination at baseline, and POMS depressive mood at baseline. For HRV at baseline a non-parametric Mann-Whitney test was performed (non-normality of the data). No significant differences were found between the conditions at baseline, all $p \mathrm{~s}>.05$, and gender was evenly distributed as well, $\chi^{2}(1, N=37)=0.17, p=.68, \varphi_{c}=.07$. To test for pre-existing differences on PANAS trait affectivity, RRS trait rumination, BDI depressive symptoms, and STAI trait anxiety, we performed a multivariate ANOVA with thinking style condition as betweensubjects variable given the interrelations between these questionnaire measures. Some variables were transformed with a logarithmic transformation before analysis (STAI-trait, RRS reflection, and PANAS negative trait affectivity). A marginally significant effect of condition was found, $F(7,29)=2.33, p=.05, \eta_{p}{ }^{2}=.36$. Univariate ANOVAs showed a 
significant difference between conditions only on STAI trait anxiety ${ }^{4}, F(1,35)=4.29, p=$ $.05, \eta_{p}^{2}=.11$

Thinking-style manipulation check. The effect of the manipulation on state rumination was investigated in a mixed ANOVA, with Time (baseline, post-induction) as a within-subjects factor, thinking style (rumination vs. problem-solving) as the betweensubjects factor, and the scores on the $\mathrm{MRSI}^{5}$ as dependent variable. Results from the mixed ANOVA on state rumination showed a significant main effect of time, $F(1,34)=53.62, p<$ $.01, \eta_{p}{ }^{2}=.61$, and a significant Time $\mathrm{x}$ Thinking Style interaction, $F(1,34)=8.39, p=.01, \eta_{p}^{2}$ $=.20$. When investigating the effect on state rumination, we also controlled (as a covariate) for the change in negative mood across the induction, assessed with the POMS depression scale, but results remained similar.

To follow-up on the Time $\mathrm{x}$ Thinking Style interaction we performed repeated measures ANOVAs with time (baseline, post-induction) as within-subjects variable for each induction condition separately. These analyses revealed that the rumination condition showed a significant increase in state rumination from baseline $(M=19.11, S D=5.40)$ to postinduction $(M=27.53, S D=5.27), F(1,18)=80.14, p<.01, \eta_{p}^{2}=.82$, also when taking into account the change in depressive mood across the induction, $F(1,17)=29.33, p<.01, \eta_{p}{ }^{2}=$ .63. The problem-solving group showed a significant increase in state rumination from baseline $(M=21.88, S D=6.62)$ to post-induction $(M=25.53, S D=7.98)$ as well, $F(1,16)=$ $6.88, p=.02, \eta_{p}^{2}=.30$, also when taking into account the change in depressive mood across the induction, $F(1,15)=4.56, p=.05, \eta_{p}^{2}=.23$. Importantly however, the Time $\mathrm{x}$ Thinking Style interaction indicates that the increase in state rumination is significantly larger in the rumination condition than in the problem-solving condition.

\footnotetext{
${ }^{4}$ Including STAI trait anxiety as a covariate in following analyses had no influence on the results.

${ }^{5}$ Data for baseline MRSI was missing for one participant from the problem-solving condition.
} 
We next conducted a univariate ANOVA with thinking style as the between-subjects factor on the logarithmically transformed HRV index measured during the thinking style induction as the dependent variable. This analysis revealed a near significant effect of thinking style, $F(1,31)=3.92, p=.06, \eta_{p}{ }^{2}=.11^{6}$. The rumination condition tended to show lower scores on the RMSSD index of HRV during the thinking style manipulation (untransformed $M=41.70, S D=24.75$ ) than the problem-solving condition (untransformed $M$ $=60.72, S D=35.75)^{7}$

Rumination and attentional breadth for self-related information. We performed a mixed ANOVA with word (ME vs. LR) and distance (close vs. far) as within-subjects factors and thinking style (rumination vs. problem-solving) as between-subjects factor with accuracy rates on the peripheral task as dependent variable (i.e. the proportions of correctly localized target stimuli in the attentional breadth task). Table 2 shows the means and standard deviations for the proportions of correctly localized target stimuli for each type of trial. This analysis yielded only a significant main effect of distance, $F(1,35)=130.41, p<.01, \eta_{p}^{2}=$ .79 , indicating that in general participants were better in localizing close targets as compared to far targets (all other $F \mathrm{~s}<2.5 ; p \mathrm{~s}>.10 ; \eta_{p}{ }^{2} \mathrm{~s}<.06$ ). Taking into account, as a covariate, the change in POMS depressive mood across the induction, did not change these results.

Additional analyses on the direct relation between heart rate variability during the thinking style induction and attentional breadth for self-related information relative to other-related information can be found in the Supplemental Material (S1) available online.

Moderation influences. We investigated whether trait rumination (the RRS) or depressive symptoms (the BDI-II-NL) qualified the effect of induction condition on

\footnotetext{
${ }^{6}$ Heart rate data was missing for 2 participants from the rumination condition and 1 participant from the problem-solving condition, due to error in measurement with the telemetric heart rate monitor. Result after removing a multivariate outlier as indicated by standardized residuals $>2.5 \mathrm{SD}$.

${ }^{7}$ Additionally we examined whether trait rumination, or the presence of depressive symptoms qualified sensitivity to the rumination or problem solving induction. However, neither trait rumination (i.e. the RRS total and subscale scores) nor depressive symptoms (i.e. the BDI-II-NL) moderated the effect of the thinking-style manipulation on state rumination or heart rate variability.
} 
attentional breadth for self-related information contrasted to other-related information. First, hierarchical multiple regression analyses were performed to test for moderation effects, following the approach of Aiken and West (1991), with a separate analysis for each moderator. The scores of the RRS (total score and subscale scores) and BDI-II-NL were centered and subsequently the interactions were calculated with the variable thinking style. In the regression analysis, thinking style $(0=$ problem-solving, $1=$ rumination $)$ and the moderator variable were entered as predictors in a first step and the interaction term was entered as a predictor in the second step of the analysis (separately for each moderator variable). The calculated $\Delta \mathrm{ANI}$ score, which represents attentional breadth for self-related information relative to other-related information, was entered as the dependent variable.

Results from the moderation models revealed that the presence of depressive symptoms did not moderate the effect of induction condition on attentional breadth. The first model without the interaction term, was not significant, $F(2,34)=0.31, p=.73, R^{2}=.02$, the second model including the interaction term was not significant either, $F(3,33)=0.23, p=$ $.87, R^{2}=.02$, nor was there a significant change in explained variance, $F$-change $(1,33)=0.09$, $p=.77, \Delta R^{2}<.01$. Furthermore, the interaction term between thinking style and BDI-II-NL was not significant, $t=0.30, p=.77$. However, the RRS total score moderated the relation ${ }^{8}$. The first model without the interaction term, was not significant, $F(2,33)=0.09, p=.92, R^{2}=$ .01 , the second model including the interaction term was marginally significant, $F(3,32)=$ 2.36, $p=.09, R^{2}=.02$, and there was a significant change in explained variance, $F$ change $(1,32)=6.87, p=.01, \Delta R^{2}=.18$. Furthermore, the interaction term between thinking style and RRS total score was significant, $t=2.62, p=.01$. When the change in POMS depressive mood across the induction was also entered in the first step of the hierarchical

\footnotetext{
${ }^{8}$ Results are reported after removal of an influential case, indicated by DfFit value $>1$. Analysis including this influential case yielded a marginal significant interaction term, $t=-1.86, p=.07, \Delta R^{2}=$ .10. Moreover, this moderation effect of the RRS total score did not seem to be driven by any of the
} 
regression analysis to take into account the change in depressive mood across the induction, results remained similar.

To better understand the direction of the moderation effect of the RRS total score, we estimated different conditional effects of thinking style on $\triangle \mathrm{ANI}$ at low (one SD below the mean), moderate (sample mean), and high (one SD above the mean) values of the moderator (i.e. the RRS score), using Hayes and Matthes' SPSS macro (Hayes \& Matthes, 2009).

Results of this simple slope analysis showed a positive relation between thinking style and the $\Delta$ ANI score at high levels of trait rumination, $t(34)=2.10, p=.044, b=0.13$, whereas such a relation was non-significant at moderate levels, $t(34)=0.32, p=.752, b=0.01$, and at low levels of trait rumination, $t(34)=-1.64, p=.111, b=-0.10$. This outcome indicates that at high levels of trait rumination induced rumination, compared to the problem-solving condition, showed more attentional narrowing for self-related information in comparison to other-related information. Additional analyses which can be found in the Supplemental Material (S2) available online, showed that this was driven by a qualified effect (by RRS) of induction condition on accuracy for localizing far target stimuli when self-related information was presented as compared to other-related information.

\section{Relation between questionnaire measures and attentional breadth for self-related}

information. We further examined the relations between (trait) characteristics assessed with the questionnaires and attentional breadth for self-related information relative to other-related information (i.e. $\triangle \mathrm{ANI})$. However, no significant correlations were observed, all $r$ s <.30; $p$ s > .05 , and results were similar after controlling (with partial correlations) for the change in MRSI state rumination or POMS depressive mood across the thinking-style manipulation.

specific subscales of the RRS (i.e. reflection and brooding) as these did not significantly moderate the effect of induction condition on attentional breadth (all $p \mathrm{~s}>.05 ; \Delta R^{2} \mathrm{~s}<.04$ ). 


\section{Discussion}

The aim of this experiment was to investigate the relation between the style of thinking in response to a stressful self-related event and visuospatial attentional breadth for self-related information, compared to information that is not related to the self. We first investigated the effectiveness of our new developed rumination versus problem-solving induction on a measure of state rumination and heart rate variability. Results from the manipulation check revealed that the adjusted induction scenario (after the pilot study, see footnote 3 ) increased the effectiveness. This was shown by a significant difference between induction conditions in the increase in state rumination, as well as a marginally significant difference between conditions in heart rate variability during the thinking-style manipulation. The rumination condition, compared to the problem-solving condition, showed lower heart rate variability, which has previously been associated with the experience of stress and worry (Pieper et al., 2007).

The main aim was to test the prediction that thinking style influences attentional breadth for self-related contrasted to not-self-related information. Results from this experiment failed to show evidence for a direct relation between thinking style and attentional breadth. However, moderation analyses revealed that trait rumination qualified this relation. Results showed that at high levels of trait rumination, momentary induced rumination as compared to problem-solving was related to a more narrow attentional scope for self-related information relative to other-related information. Follow-up analyses (see supplemental material available online S2) showed that this was reflected in the rumination condition being less accurate in localizing far target stimuli when self-related information was presented as compared to other-related information, but only at high levels of trait rumination as this was reversed at low levels of rumination. This finding suggests that individuals who have the 
tendency to ruminate in daily life are the ones who show the narrowing effects from ruminative thinking on subsequent attentional scope for self-related information.

Importantly, the effects of the thinking-style manipulation on attentional breadth at high levels of trait rumination remained significant when taking into account induction-related changes in depressive mood. Whitmer and Gotlib (2013) proposed negative mood to narrow attention, increasing the likelihood of rumination which in turn exacerbates negative mood leading to more attentional narrowing and more repetitive thoughts. However, our results indicate that a more ruminative way of thinking by itself can already be associated with a more narrowed attentional scope for self-related information relative to other-related information - at high trait rumination levels - and that these attentional effects are not fully explained by the relation of rumination with negative mood.

Examination of the relationships between (trait) characteristics and attentional breadth revealed no significant relations. However, examining the relation between trait characteristics and attentional breadth may have been influenced by the thinking-style manipulation that could obscure the relation between trait characteristics and attentional breadth. Furthermore, the attentional scope model (Whitmer \& Gotlib, 2013) proposed that trait ruminators in general show a more narrowed attentional scope. Therefore, in a second experiment we further investigated the direct relation between trait characteristics and attentional breadth for self-related information relative to other-related information in the absence of a thinking-style manipulation.

\section{Experiment 2}

\section{Method}

Twenty-seven undergraduates (22 females) aged between 17 and 45 years $(M=23.15$, $S D=5.31)$ participated in this experiment and were paid $€ 5$. This experiment was approved by the local ethical committee of the Faculty of Psychology at Ghent University. 
The questionnaires and attentional-breadth task from the previous experiment were used in this one. After informed consent, participants filled out the trait questionnaires, the POMS, and MRSI, and then they performed the attentional breadth task.

\section{Results}

Preliminary analyses. The data from one participant were deleted because in more than $50 \%$ of self-trials the central stimulus was incorrectly identified. In the remaining sample an average of $2.92 \%$ of the trials was deleted because of incorrectly identifying the central stimulus. Due to non-normality of the data a nonparametric Kruskal-Wallis test was performed to test for differences among the three groups (i.e. rumination condition experiment 1 , problem-solving condition - experiment 1 , current sample). No group related differences were found in terms of the percentages of trials that were deleted, $H(2)=0.31, p=$ .86.

Participant characteristics. Means and standard deviations for scores on the questionnaires and baseline mood measures in the current sample are shown in Table 1. To investigate whether this sample was comparable to the sample from the first experiment we tested for pre-existing differences among the three groups. We performed ANOVAs with group (rumination condition from experiment 1, problem-solving condition from experiment 1, current sample) as a between-subjects factor and age, POMS depressive mood at baseline, and MRSI state rumination at baseline as separate dependent measures. No group differences were found on age and POMS depressive mood, all $p \mathrm{~s}>.05 ; \eta_{p}{ }^{2} \mathrm{~s}<.07$. However, a significant effect of group was found for MRSI state rumination, $F(2,59)=4.19, p=.02, \eta_{p}^{2}=$ .12; Gabriel post-hoc tests revealed that the current sample reported significantly higher MRSI state rumination scores than the rumination condition from experiment $1, p=.02$. Gender was evenly distributed, $\chi^{2}(2, N=63)=0.53, p=.77, \varphi_{c}=.09$. To test for pre-existing differences on PANAS trait affectivity, RRS trait rumination, BDI depressive symptoms, and 
STAI trait anxiety, we performed a multivariate ANOVA with group as a between-subjects factor, given the interrelations between these questionnaire measures. No significant effect of group was found, Pillai's Trace $F(14,110)=1.02, p=.44, \eta_{p}^{2}=.12$.

Relation between questionnaire measures and attentional breadth. We investigated the relations between trait characteristics as measured with the questionnaires and attentional breadth. We performed a mixed ANOVA with word (ME vs. LR) and distance (close vs. far) as within-subjects factors and the questionnaire scores as continuous independent variable (i.e. covariate), for each questionnaire separately. Accuracy rates on the peripheral task were entered as dependent variable (i.e. the proportions of correctly localized target stimuli in the attentional breadth task). Table 2 shows the means and standard deviations for the proportions of correctly localized target stimuli for each type of trial. Results from the analyses with RRS total revealed a significant main effect of distance, $F(1,24)=8.96, p=.01, \eta_{p}^{2}=.27$, and the Word $\mathrm{x}$ Distance $\mathrm{x}$ RRS total interaction was marginally significant, $F(1,24)=3.27, p=.08, \eta_{p}^{2}=.12$. Although, the RRS reflection subscale had no significant effect on attentional breadth, the mixed ANOVA revealed only a main effect of distance, $F(1,24)=8.46, p=.01, \eta_{p}{ }^{2}=.26$, RRS brooding was significantly related to attentional breadth for self-related as compared to other-related information. There was a significant main effect of distance, $F(1,24)=6.44, p=.02, \eta_{p}^{2}=.21$, a marginally significant Word $\mathrm{x}$ Distance interaction, $F(1,24)=3.63, p=.07, \eta_{p}^{2}=.13$, but the Word $\mathrm{x}$ Distance $\mathrm{x}$ RRS brooding interaction was significant, $F(1,24)=4.80, p=.04, \eta_{p}{ }^{2}=.17$. To follow-up this significant Word x Distance x RRS brooding interaction we calculated the correlation between RRS brooding and the $\Delta$ ANI score, $r=.41, p=.04$, which showed that higher brooding scores were related to more attentional narrowing for self-related information relative to other-related information. Figure 2 graphically displays the relation between RRS brooding and the $\Delta$ ANI score. Additional analyses which can be found in the Supplemental 
Material (S3) available online, showed that this was driven by a relation between brooding and lower accuracy scores for localizing far target stimuli when self-related information was presented as compared to other-related information.

\section{Discussion}

The aim of the second experiment was to investigate the relations between (trait) characteristics and attentional breadth when no thinking style induction was performed. Results from this experiment revealed that higher levels of trait brooding are associated with more attentional narrowing for self- compared with other-related information. Follow-up analyses (see supplemental material available online S3) showed that this was reflected in brooding being related to lower accuracy scores for localizing far target stimuli when selfrelated information was presented in comparison to other-related information. These findings are in line with predictions from the attentional scope model of rumination (Whitmer \& Gotlib, 2013).

\section{General Discussion}

The main aim of this series of experiments was to investigate the relationship between thinking style and visuospatial attentional breadth for self-related information, based on the proposed association of (trait) rumination with a more narrow attentional scope (Whitmer \& Gotlib, 2013). Based on the attentional scope model, we hypothesized that the induced ruminative way of thinking, compared to a problem-solving approach, would be related to a more narrowed attentional scope for self-related information relative to other-related information.

In the first experiment we investigated the effects of a rumination induction, as compared to a problem-solving induction, on attentional narrowing for self- compared with other-related information and whether this effect would be qualified by trait rumination or the presence of depressive symptoms. The thinking-style manipulation check indicated that the 
rumination condition reported a significant greater increase in state rumination than the problem-solving condition, and the rumination condition tended to show lower heart rate variability than the problem-solving condition. We observed an effect of thinking style on attentional breadth for self-related contrasted to other-related information, but this effect was qualified by trait rumination. The current findings suggest that individuals who have a stronger tendency to ruminate in daily life and have recently been induced to do so are the ones who show a more narrowed attentional scope for self-related relative to other-related information. Interestingly, this effect of thinking style on attentional breadth could not be explained by individual differences in the change in depressive mood across the induction. Although the attentional scope model of rumination (Whitmer \& Gotlib, 2013) proposes that increases in negative mood lead to attentional narrowing which then increases the likelihood of thoughts becoming repetitive, our results suggest that mood does not play a role.

In the second experiment we examined the relation between trait characteristics and attentional breadth for self-related information, without a thinking-style manipulation that could influence this relation. Based on the attentional scope model (Whitmer \& Gotlib, 2013), we hypothesized that higher trait rumination scores would be related to more attentional narrowing for self- compared with other-related information. Indeed we observed that higher trait rumination scores, specifically trait brooding (considered to be the more maladaptive form of rumination), were associated with more narrowed attention for self- compared with other-related information.

The presented experiments are the first to directly test the influence of induced rumination and trait rumination on visuo-spatial attentional breadth for self-related information. The predictions of the attentional scope model (Whitmer \& Gotlib, 2013) about the relation between trait rumination and a more narrowed attentional scope were mostly based on evidence for attentional narrowing at the level of working memory processes. Our 
results, however, show that rumination interacts with lower-level cognition, as shown by an influence on the visuo-spatial scope of attention. The results indicate that trait ruminators generally show more narrowed attention for self-related information as compared to otherrelated information. It is possible that people who generally show more narrowed attention for self-related information relative to other-related information are more likely to ruminate in response to distress and that in this way they develop a tendency to ruminate over time. Nevertheless, the reverse relationship is just as likely, that high trait ruminators may have elaborated more on- and spend more time at thinking about self-related information in a ruminative manner, which over time could have resulted in generally more narrowed attention for self-related information as compared to other-related information. This seems plausible given our finding that in people with a tendency to ruminate, as compared to problem-solving, momentary ruminative thinking resulted in more narrowed attention for self-related information relative to other-related information.

The finding that at high levels of trait rumination, induced rumination as compared to problem-solving was related to more narrow attention for self-related information in comparison to other-related information, could also explain the self-perpetuating nature of this mode of processing. That is, in people with a prior tendency to ruminate, these effects of momentary ruminative thinking on external attention when confronted with self-related as compared to other-related information could further reduce the likelihood that thoughts shift away from one's feelings and problems once activated in mind. This pattern could also characterize the cognitive contribution of rumination to a worsening or maintaining of depressive mood.

The current study examined visuo-spatial attention whereas several authors have argued that rumination is largely verbal in nature and could be more directly related to conceptual breadth of attention. By design, we only tested the effect of a thinking-style 
manipulation on attentional breadth for self-related information, whereas a more narrowed attentional scope might also increase the occurrence of rumination, consistent with the attentional scope model of rumination (Whitmer \& Gotlib, 2013). Clearly, this was a first effort in testing direction of causality, and future research should also explore how manipulating the attentional scope affects the probability of state rumination.

Some limitations deserve mentioning. A newly developed rumination induction was contrasted to an active problem-solving approach. Although the manipulation successfully differentiated state-rumination scores according to the two induction conditions, state rumination also increased in the problem-solving condition, possible due to the very negative nature of the scenario. The current scenario is likely to increase feelings of guilt which is an important theme and symptom in depression; however, the situation of a car accident may also be related to anxiety. Future research might therefore use a different scenario focusing more on loss, guilt, and symptoms of depression to more specifically target depressive rumination. The ideal choice for a scenario would be one that is negative enough to facilitate rumination in an unselected sample following ruminative instructions, but ambiguous enough to sidestep rumination following problem-solving instructions.

The current study paves the way for new research in this area. First, because the manipulation of state rumination permitted causal inferences about performance in the ensuing attention task. the use of other attention tasks in subsequent experiments can provide boundary conditions. For example, according to the attentional scope model, ruminators should not become unusually distracted by peripheral self-related information as they focus attention on a typical self-irrelevant task. Another avenue to pursue is the nature of the transition from the internal self-focus of rumination toward a sensitivity to external referents to that focus. It has been argued that rumination is characterized by an internal focus on conceptual, self-related themes (Nolen-Hoeksema et al., 2008; Whitmer \& Gotlib, 2013). 
Understanding how such a focus becomes oriented toward external representations of self is important. For example, does the sensitivity develop over a series of similar trials, such as those presented in our experiments, or can it appear without practice if the external event is sufficiently personal? Third, there are recent efforts to modulate rumination via training or cognitive bias modification procedures (e.g. Cohen, Mor \& Henik, 2014; Hertel, Mor, Ferrari, Hunt, \& Agrawal, 2014). Another possible direction for future work therefore concerns the modification of attentional scope and the subsequent examination of ruminative tendencies. To modify the scope of visual attention, researchers might use extant training procedures (e.g. Gable \& Harmon-Jones, 2012), adjusted by the inclusion of self-relevant (or emotional) content. Pursuing any of these issues could further our understanding of attentional effects of rumination as one element of our understanding of rumination more generally, as well as its contribution to recurrent patterns of depression (Nolen-Hoeksema, 2000). Each processanalytic element of that understanding can then be incorporated into application to treatment methods or set aside as a direction not to pursue. 


\section{Author's contribution}

All authors contributed to the development of the study concept and study design.

Testing and data collection was performed by M.G. at Ghent University, Belgium. M.G.

performed the data analysis and interpretation under the supervision of R.D.R. M.G. drafted the paper and P.H., E.H.W.K. and R.D.R provided critical revisions. All authors approved the final version of the paper for submission. 


\section{Acknowledgements}

This research was supported by Grant BOF10/GOA/014 for a Concerted Research Action of Ghent University awarded to Rudi De Raedt and Ernst Koster.

We thank Marleen Van Roy for her valuable help in testing the participants, and collecting the data for the second experiment. 


\section{References}

Aiken, L.S., \& West, S. G. (1991). Multiple regression: Testing and interpreting interactions. Newbury Park: Sage.

Ayduk, O., \& Kross, E. (2010). From a distance: Implications of spontaneous self-distancing for adaptive self-reflection. Journal of Personality and Social Psychology, 98, 809829. doi: $10.1037 / \mathrm{a} 0019205$

Ball, K. K., Beard, B. L., Roenker, D. L., Miller, R. L., \& Griggs, D. S. (1988). Age and sisual-search: Expanding the useful field of view. Journal of the Optical Society of America A-Optics Image Science and Vision, 5, 2210-2219. doi: 10.1364/JOSAA.5.002210

Beck, A. T., Steer, R. A., \& Brown, G. K. (1996). BDI-II manual. San Antonio, TX: The Psychological Corporation.

Bosmans, G., Braet, C., Koster, E., \& De Raedt, R. (2009). Attachment security and attentional breadth toward the attachment figure in middle childhood. Journal of Clinical Child and Adolescent Psychology, 38, 872-882. doi: $10.1080 / 15374410903258926$

Cohen, N., Mor, N., \& Henik A. (2014). Linking executive control and emotional response: A training procedure to reduce rumination. Clinical Psychological Science. doi: $10.1177 / 2167702614530114$

Cottyn, J., De Clercq, D., Pannier, J.-L., Crombez, G., \& Lenoir, M. (2006). The measurement of competitive anxiety during balance beam performance in gymnasts. Journal of sports sciences, 24, 157-164. doi:10.1080/02640410500131571

Derryberry, D., \& Tucker, D.M. (1994). Motivating the focus of attention. In P.M. Niedenthal, S. Kitayama, S (Eds.), The heart's eye: Emotional influences in perception and attention (pp.167-196). San Diego, CA: Academic Press. 
Fredrickson, B.L., \& Branigan, C. (2005). Positive emotions broaden the scope of attention and thought-action repertoires. Cognition \& Emotion, 19, 313-332. doi: $10.1080 / 02699930441000238$

Gable, P.A., \& Harmon-Jones, E. (2012). Reducing attentional capture of emotion by broadening attention: Increased global attention reduces early electrophysiological responses to negative stimuli. Biological Psychology, 90, 150-153. doi: 10.1016/j.biopsycho.2012.02.006

Grol, M., Koster, E. H. W., Bruyneel, L., \& De Raedt, R. (2014). Effects of positive mood on attention broadening for self-related information. Psychological Research, 78, 566573. doi: 10.1007/s00426-013-0508-6.

Hayes, A.F., \& Matthes, J. (2009). Computational procedures for probing interactions in OLS and logistic regression: SPSS and SAS implementations. Behavior Research Methods, 41, 924-936. doi: 10.3758/BRM.41.3.924

Hertel, P. T., Mor, N., Ferrari, C., Hunt, O., \& Agrawal, N. (2014). Looking on the dark side: Rumination and cognitive bias modification. Clinical Psychological Science.

Karpinski, A., \& Steinman, R. B. (2006). The single category Implicit Association Test as a measure of implicit social cognition. Journal of Personality and Social Psychology, 91, 16-32. doi: 10.1037/0022-3514.91.1.16

Kleiger, R.E., Stein, P.K., Bosner, M.S., \& Rottman, J.N. (1992). Time domain measurements of HRV. Cardiology Clinical, 10, 487-498.

Kross, E., \& Ayduk, O. (2011). Making meaning out of negative experiences by selfdistancing. Current Directions in Psychological Science, 20, 187-191. doi: $10.1177 / 0963721411408883$ 
Lyubomirsky, S., \& Nolen-Hoeksema, S. (1995). Effects of self-focused rumination on negative thinking and interpersonal problem-solving. Journal of Personality and Social Psychology, 69, 176-190. doi: 10.1037/0022-3514.69.1.176

McNair, D.M., Lorr, M., \& Droppelman, L.F. (1971). Manual for the profile of mood states. San Diego, CA: Educational and Industrial Testing Services.

Mor, N., Marchetti, I., \& Koster, E. H. W. (2013). A new state measure of self-reflection: Psychometric evaluation of the Momentary Ruminative Self-focus Inventory (MRSI). Manuscript in preparation.

Mor, N., \& Winquist, J. (2002). Self-focused attention and negative affect: A meta-analysis. Psychological Bulletin, 128, 638-662. doi: 10.1037/0033-2909.128.4.638

Niskanen, J.-P., Tarvainen, M. P., Ranta-Aho, P. O., \& Karjalainen, P. A. (2004). Software for advanced HRV analysis. Computer methods and programs in biomedicine, 76, 73 81. doi:10.1016/j.cmpb.2004.03.004

Nolen-Hoeksema, S. (2000). The role of rumination in depressive disorders and mixed anxiety/depressive symptoms. Journal of Abnormal Psychology, 109, 504-511. doi: 10.1037/0021-843X.109.3.504

Nolen-Hoeksema, S., \& Morrow, J. (1991). A prospective study of depression and posttraumatic stress symptoms after a natural disaster: The 1989 Loma Prieta earthquake. Journal of Personality and Social Psychology, 61, 115-121. doi: 10.1037/0022-3514.61.1.115

Nolen-Hoeksema, S., Wisco, B.E., \& Lyubomirsky, S. (2008). Rethinking rumination. Perspectives on Psychological Science,3, 400-424. doi: 10.1111/j.17456924.2008.00088.x 
Pieper, S., Brosschot, J. F., van der Leeden, R., \& Thayer, J. F. (2007). Cardiac effects of momentary assessed worry episodes and stressful events. Psychosomatic medicine, 69, 901-909. doi:10.1097/PSY.0b013e31815a9230

Portzky, M. (2008). RS-nl Resilience Scale-Nederlandse versie. Amsterdam: Harcourt Publishers, 25-50.

Raes, F., Hermans, D., \& Eelen, P. (2003). De Nederlandstalige versie van de Ruminative Response Scale en de Rumination on Sadness Scale (The Dutch version of the Rumination Response Scale and the Rumination on Sadness Scale). Gedragstherapie, $36,97-104$.

Segal, Z.V., Kennedy, S., Gemar, M., Hood, K., Pedersen, R., \& Buis, T. (2006). Cognitive reactivity to sad mood provocation and the prediction of depressive relapse. Archives of General Psychiatry, 63, 1-7. doi: 10.1001/archpsyc.63.7.749

Spielberger, C. D., Gorsuch, R. L., Lushene, R., Vagg, P. R., \& Jacobs, G. A. (1983). Manual for the state-trait anxiety inventory. Palo Alto (CA): Consulting Psychologists Press.

Task Force of the European Society of Cardiology the North American Society of Pacing and Electrophysiology (1996). Heart Rate Variability : Standards of Measurement, Physiological Interpretation, and Clinical Use. Circulation, 93(5), 1043-1065. doi: 10.1161/01.cir.93.5.1043

Treynor, W., Gonzalez, R., \& Nolen-Hoeksema, S. (2003). Rumination reconsidered: A psychometric analysis. Cognitive Therapy and Research, 27, 247-259. doi: 10.1023/A:1023910315561

Van der Does, A. J.W. (2002). De Nederlandse versie van de Beck Depression Inventory. Tweede Editie (2nd ed.). In: The Dutch version of the Beck Depression Inventory Lisse: Swets \& Zeitlinger b.v. 
Van der Ploeg, H. M., Defares, P. B., \& Spielberger, C. D. (2000). Handleiding bij de ZelfBeoordelings Vragenlijst. Een Nederlandstalige bewerking van de Spielberger StateTrait Anxiety Inventory. [Manual for the State-Trait Anxiety Inventory]. Swets and Zeitlinger b.v., Lisse.

Wald, F. (1984). De verkorte POMS [The shortened POMS]. Amsterdam: Onuitgegeven doctoraalwerk, vakgroep psychonomie, psychologisch laboratorium, Universiteit van Amsterdam.

Watkins, E.R. (2008). Constructive and unconstructive repetitive thought. Psychological Bulletin, 184, 163-206. doi: 10.1037/0033-2909.134.2.163

Watson, D., Clark, L. A., \& Tellegen, A. (1988). Development and Validation of Brief Measures of Positive and Negative Affect - the Panas Scales. Journal of Personality and Social Psychology, 54, 1063-1070. doi: 10.1037/0022-3514.54.6.1063

Whitmer, A.J. \& Gotlib, I.H. (2013). An attentional scope model of rumination. Psychological Bulletin, 139, 1036-1061. doi: 10.1037/a0030923 
Table 1

Participant characteristics

\begin{tabular}{lccc}
\hline & Rumination $(n=19)$ & Problem-solving $(n=18)$ & No induction $(n=26)$ \\
& $M(S D)$ & $M(S D)$ & $M(S D)$ \\
\hline Age & $21.21(1.96)$ & $21.33(2.11)$ & $23.19(5.41)$ \\
Gender & 16 females & 16 females & 22 females \\
PANAS trait positive & $34.37(5.68)$ & $32.78(7.65)$ & $34.38(5.46)$ \\
PANAS trait negative & $17.24(4.61)$ & $17.94(7.33)$ & $17.35(5.61)$ \\
RRS total & $43.00(11.15)$ & $45.22(11.37)$ & $45.08(10.40)$ \\
RRS reflection & $8.84(2.34)$ & $9.78(3.86)$ & $10.85(2.90)$ \\
RRS brooding & $10.53(3.75)$ & $11.00(3.45)$ & $24.27(5.78)$ \\
MRSI baseline & $19.11(5.41)$ & $21.88(6.62)$ & $7.58(7.89)$ \\
BDI & $4.26(4.70)$ & $8.11(8.17)$ & $3.12(6.47)$ \\
POMS depression baseline & $1.00(2.73)$ & $1.83(3.60)$ & $40.88(11.80)$ \\
STAI-trait & $36.95(7.80)$ & $43.78(11.54)$ & n.a. \\
HRV baseline & $42.09(25.35)$ & $59.54(35.63)$ & \\
\hline
\end{tabular}


Table 2

Proportion of correctly localized target stimuli for each type of trial

\begin{tabular}{llllclc}
\hline & \multicolumn{2}{c}{ Rumination } & \multicolumn{2}{c}{ Problem-solving } & \multicolumn{2}{c}{ No induction } \\
& \multicolumn{2}{c}{$M(S D)$} & \multicolumn{2}{c}{$M(S D)$} & \multicolumn{2}{c}{$M(S D)$} \\
& Word: & Word: & Word: & Word: & Word: & Word: \\
& "ME" & "LR" & "ME" & "LR" & "ME" & "LR" \\
\hline Distance: close & $.92(.11)$ & $.91(.11)$ & $.90(.09)$ & $.89(.10)$ & $.90(.11)$ & $.88(.10)$ \\
Distance: far & $.60(.18)$ & $.61(.21)$ & $.50(.27)$ & $.50(.28)$ & $.64(.17)$ & $.65(.17)$ \\
\hline
\end{tabular}




\section{Figure Captions}

Figure 1. Illustration of a trial in the Attentional Breadth Task. The gray dots are presented in pairs of two, simultaneously with the central word and the smaller black circle. The first response screen asks participants which word they have seen. The second response screen asks participants on which of eight axes the black circle was presented.

Figure 2. Graphical display of the relation between RRS brooding and the $\Delta$ ANI score. 
Figure 1.
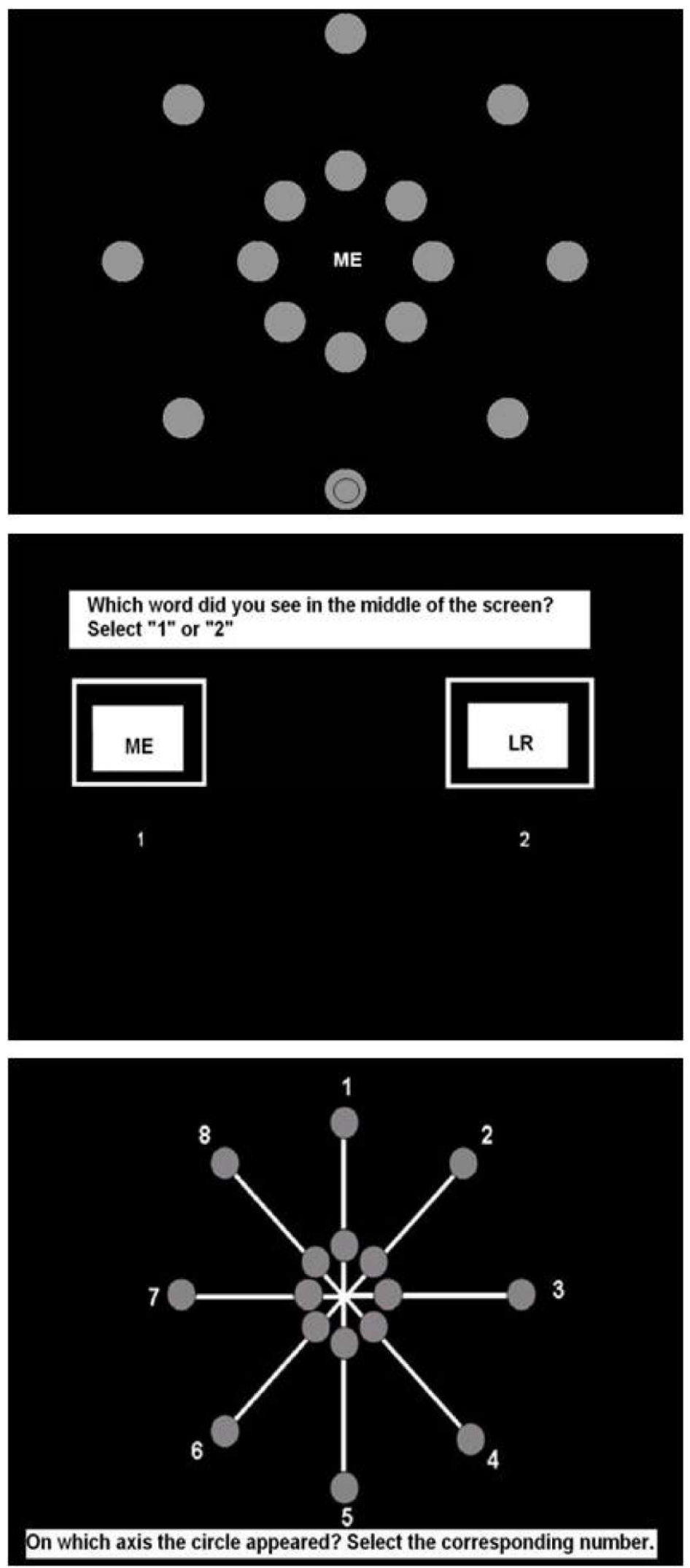
Figure 2.

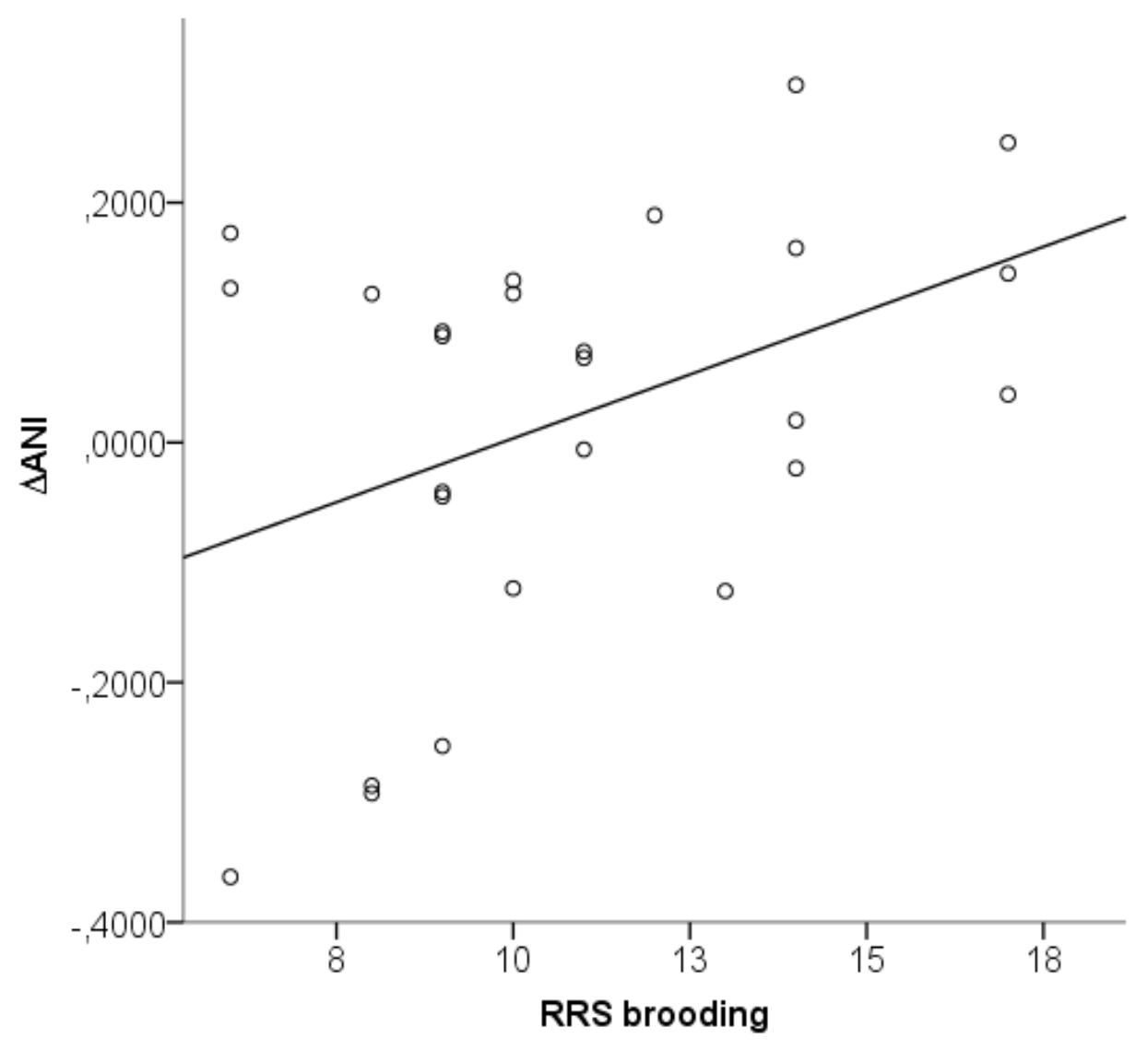

